\title{
A model of weakly vortical interfacial flow
}

\author{
G. A. Jarrad* A. J. Roberts ${ }^{\dagger}$
}

(Received 31 January 2000)

\begin{abstract}
A boundary-integral model is derived to describe two-dimensional, weakly viscous, quasi-irrotational fluid flow at the interface between two fluids. Vortical flow is restricted to a thin boundary layer, allowing the interfacial fluid dynamics to be largely generated by a vortex sheet approximation. A theoretical analysis of the model reveals that
\end{abstract}

*Dept of Mathematics, University of S.A., Mawson Lakes, South Australia, 5095. mailto:Geoff. Jarrad@cmis.csiro.au

${ }^{\dagger}$ Dept of Maths and Computing, University of Southern Qld, Toowoomba, Queensland, 4350, AustraliA. mailto: aroberts@usq.edu.au

${ }^{0}$ See http: //anziamj . austms . org.au/V42/E028 for this article and ancillary services,

(C) Austral. Mathematical Soc. 2001. Published 22 March 2001. 
small-amplitude interfacial waves have only conservative and dissipative modes of behaviour. In contrast, similar analyses of other models of weak vorticity reveal non-physical modes which increase in energy. Numerical simulations of our model demonstrate the appropriate conservation of key physical invariants.

\section{Contents}

1 Introduction

E71

2 Dynamics of a fluid-gas interface

E73

3 A model of weak vorticity

E75

4 Modelling the interfacial flow

E77

$5 \quad$ Stability of the weakly vortical model

E80

6 Numerical velocity calculations $\quad$ E85

6.1 Irrotational velocity calculations . . . . . . . . . . E87

6.2 Vortical velocity calculations . . . . . . . . . . E90

7 Numerical verification of the model $\quad$ E92

8 Conclusion $\quad$ E100 


\section{Introduction}

We examine two-dimensional flow on a vertical cross-section through two fluid layers. Of special interest is the behaviour of the interfacial boundary layer of the lower, denser fluid. In comparison, the upper, light fluid is assumed dynamically insignificant. A physical example of such a situation is the waves on the free surface of deep water beneath the atmosphere. The fluid dynamics are irrotational except for a thin, vortical layer at the interface. Both fluids are assumed to have infinite depth to avoid additional effects from the vorticity of the bed boundary layer. The assumption of irrotationality allows the velocity at the interface of the two fluids to be completely specified by a vortex sheet formulation (Van de Vooren [8]). A difficulty is that, on the one hand, an imposed condition of zero shear stress at the interface cannot be satisfied by purely irrotational flow unless it is also inviscid (Lundgren [3]), whereas on the other hand, the inclusion of viscosity is desirable for modelling physically realistic effects; for example, viscosity plays a significant role in chaotic wave dynamics (Holmes [2]). As a compromise, Lundgren [3] derived a quasi-irrotational, weakly viscous approximation to vortical flow, where the viscous effects appear as corrections to the time-evolution of the velocity potential. However, we show in this paper that in two dimensions Lundgren's model exhibits a non-physical instability. We derive, instead, a model which 
exhibits only conservative and dissipative modes of behaviour.

In Section 2 we consider the dynamics of an effectively incompressible, weakly viscous fluid, and we record the fundamental variables, equations and boundary conditions to be solved. In Section 3 we simplify the model by assuming that vorticity only occurs within a boundary layer near the interface. In Section 4 we focus our attention on modelling only the fluid dynamics at the interface, and derive both an Eulerian form, suitable for a theoretical investigation of stability, and a Lagrangian form, suitable for a numerical simulation of the interfacial fluid flow. In Section 5 we use the Eulerian form of the model to examine the linear stability of small-amplitude perturbations to a flat interface, and show that our model exhibits only conservative and dissipative modes of behaviour. In contrast, we also show that the models of both Lundgren [3] and Ruvinsky et al. [6] produce exponentially growing non-physical modes. In Section 6 we derive a numerical formulation for the evaluation of both the irrotational and vortical components of the fluid velocities at discrete points along the interface. The Lagrangian form of the fluid dynamical equations then forms the basis for a discrete-time-integrable numerical model. Finally, in Section 7 we use the numerical model to simulate the behaviour of small-amplitude interfacial waves. We verify that our model of weak vorticity produces physically consistent behaviour. The linear fluid dynamics are verified by comparing the numerically computed eigenvalues with those derived from perturbation analysis. The nonlinear fluid dynamics are verified by plotting the numerical values of four physically invariant quantities. 


\section{Dynamics of a fluid-gas interface}

Consider the dynamics of an effectively incompressible, weakly viscous fluid, such as water, lying below a dynamically inactive gas, such as still air. In this section, we record the fundamental variables, equations and boundary conditions to be solved. We start with the two-dimensional form of the Navier-Stokes equation of fluid motion, viz

$$
\frac{\partial \boldsymbol{v}}{\partial t}+\boldsymbol{v} \cdot \boldsymbol{\nabla} \boldsymbol{v}=\boldsymbol{g}-\frac{1}{\rho} \boldsymbol{\nabla} p+\nu \nabla^{2} \boldsymbol{v}
$$

where $\boldsymbol{v}$ denotes the fluid velocity, $\boldsymbol{g}=-g \boldsymbol{j}$ the gravitational acceleration, $\rho$ the constant density of the fluid, $p$ the fluid pressure, and $\nu$ the kinematic viscosity of the fluid. The incompressible fluid flow satisfies the continuity equation

$$
\boldsymbol{\nabla} \cdot \boldsymbol{v}=0
$$

The boundary conditions to use with these differential equations are those of continuous stress across the interface, and no motion infinitely deep in the fluid. The Newtonian fluid flow has the stress tensor

$$
\sigma=-p I+2 \rho \nu e,
$$

where $I$ denotes the identity tensor, and $e$ the rate-of-strain tensor (Batchelor [1]). By contrast, the stress within the gas is effectively

$$
\sigma_{0}=-p_{0} I
$$


for some constant pressure $p_{0}$. The imbalance of stress acting normally across the interface must be balanced by surface tension:

$$
\left(\tilde{\sigma}-\tilde{\sigma}_{0}\right) \cdot \boldsymbol{n}=\rho \varsigma \kappa \boldsymbol{n},
$$

where $\boldsymbol{n}$ is a unit vector locally normal to the interface, $\varsigma=\gamma / \rho$ is the scaled coefficient of interfacial tension, $\kappa$ is the curvature of the interface, and the symbol ' $\sim$ ' denotes evaluation at the interface.

For convenience, we define the normal straining force on the fluid at the interface as

$$
\tilde{\boldsymbol{\tau}}=\tilde{\tau} \boldsymbol{n}=\tilde{e} \cdot \boldsymbol{n}
$$

just as $\tilde{\sigma} \cdot \boldsymbol{n}$ is the corresponding normal stress. Consequently, substituting the fluid stress tensor $\sigma$ from (3) and the gas stress tensor $\sigma_{0}$ from (4) into (5) gives the pressure of the fluid at the interface as

$$
\tilde{p}=\tilde{p}_{0}+2 \rho \nu \tilde{\tau}-\rho \varsigma \kappa .
$$

Henceforth, the gas pressure $p_{0}$ is taken to be zero without loss of generality.

Deep within the fluid, the fluid motion is only slight, and diminishes further with increasing depth. The boundary condition at the hypothetical "bottom" of the infinitely deep fluid is therefore

$$
\boldsymbol{v} \rightarrow \mathbf{0} \text { as } y \rightarrow-\infty
$$

This completes the exposition of the fundamental variables, equations and boundary conditions required to specify the fluid-gas dynamical system. 


\section{A model of weak vorticity}

The model of the previous section is simplified assuming a small but nonnegligible viscosity, which acts to form weakly vortical motion only within a thin boundary layer at the interface. The fluid velocity $\boldsymbol{v}=u \boldsymbol{i}+v \boldsymbol{j}$ is written in terms of potentials $\phi=\phi(x, y, t)$ and $\psi=\psi(x, y, t)$, viz

$$
\boldsymbol{v}=\boldsymbol{v}^{i}+\boldsymbol{v}^{r}, \quad \boldsymbol{v}^{i}=\boldsymbol{\nabla} \phi, \quad \boldsymbol{v}^{r}=\boldsymbol{\nabla} \times \psi \boldsymbol{k},
$$

where $\boldsymbol{v}^{i}$ and $\boldsymbol{v}^{r}$ are the irrotational and rotational components of the velocity, respectively, and $\boldsymbol{k}$ a unit vector directed orthogonal to the plane of the fluid flow. The continuity equation (2) then reduces to

$$
\boldsymbol{\nabla} \cdot \boldsymbol{v}=\frac{\partial u}{\partial x}+\frac{\partial v}{\partial y}=\nabla^{2} \phi=0,
$$

and the vorticity of the flow is

$$
\omega=\boldsymbol{k} \cdot \boldsymbol{\nabla} \times \boldsymbol{v}=\frac{\partial v}{\partial x}-\frac{\partial u}{\partial y}=-\nabla^{2} \psi .
$$

Hence, taking both the divergence and curl of the Navier-Stokes equation (1) leads to

$$
\begin{aligned}
\nabla^{2}\left(\frac{\partial \phi}{\partial t}+\frac{1}{2} \boldsymbol{v} \cdot \boldsymbol{v}+g y+\frac{p}{\rho}\right) & =\boldsymbol{\nabla} \cdot \boldsymbol{v} \times \omega \boldsymbol{k} \\
\nabla^{2}\left(\frac{\partial \psi}{\partial t}-\nu \nabla^{2} \psi\right) & =\boldsymbol{v} \cdot \boldsymbol{\nabla} \omega
\end{aligned}
$$


respectively, where use has been made of the vector-tensor identity

$$
\boldsymbol{v} \cdot \boldsymbol{\nabla} \boldsymbol{v}=\frac{1}{2} \boldsymbol{\nabla}(\boldsymbol{v} \cdot \boldsymbol{v})-\boldsymbol{v} \times \omega \boldsymbol{k} .
$$

The right-hand sides of equations (12) and (13) are further manipulated to give

$$
\begin{aligned}
\boldsymbol{\nabla} \cdot \boldsymbol{v} \times \omega \boldsymbol{k}= & \nabla^{2}(\boldsymbol{v} \cdot \boldsymbol{\nabla} \times \omega \boldsymbol{k}-\psi \omega)+\nabla \cdot \nabla^{2} \boldsymbol{v} \times \psi \boldsymbol{k} \\
& +2 \boldsymbol{\nabla} \cdot(\boldsymbol{\nabla} \psi \cdot \boldsymbol{\nabla} \boldsymbol{v}) \times \boldsymbol{k} \\
\boldsymbol{v} \cdot \boldsymbol{\nabla} \omega= & -\nabla^{2}(\boldsymbol{v} \cdot \boldsymbol{\nabla} \psi)+(\boldsymbol{v} \cdot \boldsymbol{\nabla} \psi) \nabla^{2} \boldsymbol{v}+2 \boldsymbol{\nabla} \boldsymbol{v}: \boldsymbol{\nabla}(\boldsymbol{\nabla} \psi),
\end{aligned}
$$

where the operator ':' denotes the scalar inner-product of two tensors.

We assume the vortical part of the flow is confined to a thin boundary layer in the fluid near the interface. For a flow with length scale $L$ and a time scale $T$, the dimensionless fluid flow parameter of interest is $\delta=\sqrt{\nu T} / L$, so that the boundary layer is of depth $\mathcal{O}(\delta L)$, and the normal derivatives of vortically-related quantities are $\mathcal{O}\left(\delta^{-1}\right)$ times larger than the tangential derivatives. As a consequence, in the boundary layer $\boldsymbol{v}^{r}=\mathcal{O}(\delta L / T), \psi=$ $\mathcal{O}\left(\delta^{2} L^{2} / T\right)$ and $\omega=\mathcal{O}(1 / T)$. However, the irrotational flow is not restricted to the boundary layer, and hence $\boldsymbol{v}^{i}=\mathcal{O}(L / T)$ and $\phi=\mathcal{O}\left(L^{2} / T\right)$. A scale analysis of (15) and (16) then supports the approximation

$$
\begin{aligned}
\nabla \cdot \boldsymbol{v} \times \omega \boldsymbol{k} & \approx \nabla^{2}(\boldsymbol{v} \cdot \nabla \times \omega \boldsymbol{k}-\psi \omega) \\
\boldsymbol{v} \cdot \boldsymbol{\nabla} \omega & \approx-\nabla^{2}(\boldsymbol{v} \cdot \nabla \psi) .
\end{aligned}
$$


This formulation differs slightly from Lundgren [3] in that we have chosen, for convenience, to also neglect the isolated term $2 \frac{\partial v}{\partial y} \frac{\partial^{2} \psi}{\partial y^{2}}$ from $2 \boldsymbol{\nabla} \boldsymbol{v}: \boldsymbol{\nabla}(\boldsymbol{\nabla} \psi)$. Integrating (12) and (13) after using (17) and (18) in the right-hand sides then gives

$$
\begin{aligned}
& \frac{\partial \phi}{\partial t}=\boldsymbol{v} \cdot \boldsymbol{\nabla} \times \omega \boldsymbol{k}-\psi \omega-\frac{1}{2} \boldsymbol{v} \cdot \boldsymbol{v}-g y-\frac{p}{\rho}, \\
& \frac{\partial \psi}{\partial t}=-\boldsymbol{v} \cdot \boldsymbol{\nabla} \psi+\nu \nabla^{2} \psi,
\end{aligned}
$$

respectively, where the arbitrary constants of integration have been set to zero without loss of generality. These equations define the fluid dynamics in the boundary layer.

\section{Modelling the interfacial flow}

The boundary layer fluid dynamical model of the previous section is used to formulate both an Eulerian and a Lagrangian description of the fluid-gas interface. The Eulerian model describes temporal changes to properties of the fluid at fixed points in space, whereas the Lagrangian model describes changes to the properties of fluid "particles" which move with the fluid flow at the interface.

Let the two-dimensional interface be denoted by the curve

$$
\tilde{y}=\eta(x, t) .
$$


For the Eulerian model, the motion of the interface is obtained by restating the position-velocity relationship as the kinematic velocity relationship, viz

$$
\frac{\widetilde{D x}}{D t}=\tilde{\boldsymbol{v}} \Leftrightarrow \frac{\partial \eta}{\partial t}=\tilde{v}-\tilde{u} \frac{\partial \eta}{\partial x},
$$

where $D / D t$ represents the material derivative $\frac{\partial}{\partial t}+\boldsymbol{v} \cdot \boldsymbol{\nabla}$. Now, since the fluid pressure of the interface is given by equation (7), then equations (19) and (20) are evaluated at the interface as

$$
\begin{aligned}
\frac{\partial \phi}{\partial t} & =\frac{1}{2} \tilde{\boldsymbol{v}} \cdot \tilde{\boldsymbol{v}}-\tilde{\boldsymbol{v}} \cdot \widetilde{\nabla} \phi-\tilde{\psi} \tilde{\omega}-g \eta-2 \nu \tilde{\tau}+\varsigma \kappa, \\
\frac{\partial \psi}{\partial t} & =-\tilde{\boldsymbol{v}} \cdot \widetilde{\nabla \psi}-\nu \tilde{\omega}
\end{aligned}
$$

respectively, where use has been made of $\boldsymbol{v} \cdot \boldsymbol{\nabla} \times \omega \boldsymbol{k}=\boldsymbol{v} \cdot \boldsymbol{v}-\boldsymbol{v} \cdot \boldsymbol{\nabla} \phi$, from (9). This Eulerian model is used in the next section to investigate the stability of small-amplitude perturbations to interfacial waves.

The Lagrangian model is now obtained by reformulating the Eulerian model in terms of fundamental variables evaluated solely at the interface. Thus, for example, the chain rule for horizontal derivatives gives

$$
\begin{aligned}
& \frac{\partial \tilde{u}}{\partial x}=\frac{\widetilde{\partial u}}{\partial x}+\frac{\widetilde{\partial u}}{\partial y} \frac{\partial \eta}{\partial x}=\frac{\widetilde{\partial u}}{\partial x}+\left(\frac{\widetilde{\partial v}}{\partial x}-\tilde{\omega}\right) \frac{\partial \eta}{\partial x} \\
& \frac{\partial \tilde{v}}{\partial x}=\frac{\widetilde{\partial v}}{\partial x}+\frac{\widetilde{\partial v}}{\partial y} \frac{\partial \eta}{\partial x}=\frac{\widetilde{\partial v}}{\partial x}-\frac{\partial u}{\partial x} \frac{\partial \eta}{\partial x}
\end{aligned}
$$


from (11) and (10), respectively. By simultaneous solution, we have

$$
\begin{aligned}
& {\left[1+\left(\frac{\partial \eta}{\partial x}\right)^{2}\right] \frac{\partial u}{\partial x}=\frac{\partial \tilde{u}}{\partial x}-\frac{\partial \tilde{v}}{\partial x} \frac{\partial \eta}{\partial x}+\tilde{\omega} \frac{\partial \eta}{\partial x}} \\
& {\left[1+\left(\frac{\partial \eta}{\partial x}\right)^{2}\right] \frac{\partial v}{\partial x}=\frac{\partial \tilde{u}}{\partial x} \frac{\partial \eta}{\partial x}+\frac{\partial \tilde{v}}{\partial x}+\tilde{\omega}\left(\frac{\partial \eta}{\partial x}\right)^{2} .}
\end{aligned}
$$

Likewise, the chain rule for total temporal derivatives gives

$$
\frac{d \tilde{q}}{d t}=\frac{\widetilde{\partial q}}{\partial t}+\frac{\widetilde{\partial q}}{\partial x} \frac{d \tilde{x}}{d t}+\frac{\widetilde{\partial q}}{\partial y} \frac{d \tilde{y}}{d t}
$$

for an arbitrary quantity $q(x, y, t)$ evaluated at the interface $\tilde{y}=\eta(x, t)$. Hence, the Lagrangian model of weakly vortical interfacial dynamics is

$$
\begin{aligned}
\frac{\widetilde{D x}}{D t} & \equiv \frac{d \tilde{x}}{d t}=\tilde{u}, \\
\frac{\widetilde{D y}}{D t} & \equiv \frac{d \tilde{y}}{d t}=\tilde{v}, \\
\frac{\widetilde{D \phi}}{D t} & \equiv \frac{d \tilde{\phi}}{d t}=-g \eta+\frac{1}{2} \tilde{\boldsymbol{v}} \cdot \tilde{\boldsymbol{v}}+\varsigma \kappa-2 \nu \tilde{\tau}-\tilde{\psi} \tilde{\omega}, \\
\frac{\widetilde{D \psi}}{D t} & \equiv \frac{d \tilde{\psi}}{d t}=-\nu \tilde{\omega} .
\end{aligned}
$$

This Lagrangian model forms the basis of the numerical investigation in Section 7 of the fluid dynamics at the interface. 


\section{Stability of the weakly vortical model}

We use the Eulerian formulation of the previous section to prove that our model exhibits linear modes of behaviour which either conserve or dissipate energy. In comparison, we show that the related models of Lundgren [3] and Ruvinsky et al. [6] exhibit non-physical modes which gain energy.

The stability of a system of PDE's is primarily determined by the eigenvalues of its linearisation. These eigenvalues are obtained by an analysis of small-amplitude perturbations to the flat interface. Let the small quantity $\epsilon \ll 1$ be representative of the size of the perturbation, such that $\eta, \phi$, $\psi, u$ and $v$ are $\mathcal{O}(\epsilon)$. Now, for an arbitrary quantity $q(x, y, t)$, let $\bar{q}$ denote $q(x, 0, t)$. This allows us to express $\tilde{q}$, the value of $q$ on the interface, in terms of quantities evaluated on $y=0$, viz

$$
\tilde{q} \doteq \bar{q}+\frac{\overline{\partial q}}{\partial y} \eta+\frac{1}{2 !} \frac{\overline{\partial^{2} q}}{\partial y^{2}} \eta^{2}+\frac{1}{3 !} \frac{\overline{\partial^{3} q}}{\partial y^{3}} \eta^{3}+\cdots
$$

Thus, for example, the interfacial curvature has the expansion

$$
\kappa=\frac{\partial^{2} \eta}{\partial x^{2}}\left[1+\left(\frac{\partial \eta}{\partial x}\right)^{2}\right]^{-3 / 2} \doteq \frac{\partial^{2} \eta}{\partial x^{2}}+\mathcal{O}\left(\epsilon^{2}\right) .
$$

In order to derive equivalent expansions for $\tilde{\omega}$ and $\tilde{\tau}$, first note from (6) that the normal and tangential components of the normal straining force $\tilde{\boldsymbol{\tau}}$ are

$$
\boldsymbol{n} \cdot \tilde{e} \cdot \boldsymbol{n}=\tilde{\tau}, \quad \boldsymbol{s} \cdot \tilde{e} \cdot \boldsymbol{n}=0,
$$


respectively. The rate-of-strain tensor $e$ at the interface is

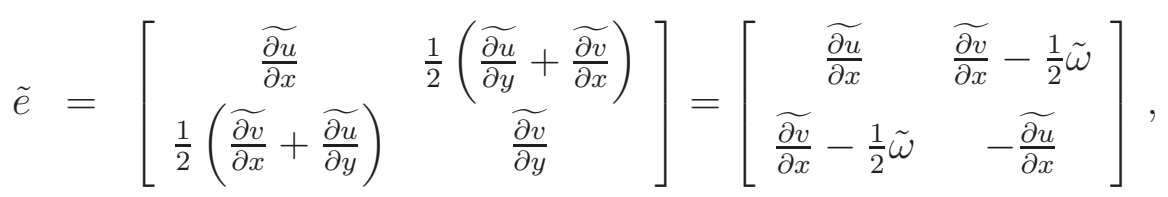

where use has been made of $\frac{\widetilde{\partial v}}{\partial y}=-\frac{\widetilde{\partial u}}{\partial x}$ from (10), and $\frac{\widetilde{\partial u}}{\partial y}=\frac{\widetilde{\partial v}}{\partial x}-\tilde{\omega}$ from (11). Hence, using relations (27) and (28), the simultaneous solutions to (36) are

$$
\begin{aligned}
\tilde{\omega} & =2\left(\frac{\partial \tilde{v}}{\partial x}-\frac{\partial \tilde{u}}{\partial x} \frac{\partial \eta}{\partial x}\right)\left[1+\left(\frac{\partial \eta}{\partial x}\right)^{2}\right]^{-1} \doteq 2 \frac{\partial v}{\partial x}+\mathcal{O}\left(\epsilon^{2}\right), \\
\tilde{\tau} & =-\left(\frac{\partial \tilde{u}}{\partial x}+\frac{\partial \tilde{v}}{\partial x} \frac{\partial \eta}{\partial x}\right) \doteq-\frac{\partial u}{\partial x}+\mathcal{O}\left(\epsilon^{2}\right) .
\end{aligned}
$$

Now, by retaining only those terms linear in $\epsilon$, the Eulerian system (22)-(24) reduces to

$$
\begin{aligned}
\frac{\partial \eta}{\partial t} & =\overline{\frac{\partial \phi}{\partial y}}-\frac{\overline{\partial \psi}}{\partial x} \\
\frac{\partial \phi}{\partial t} & =-g \eta+\varsigma \frac{\partial^{2} \eta}{\partial x^{2}}+2 \nu \frac{\overline{\partial^{2} \phi}}{\partial x^{2}}+2 \nu \frac{\overline{\partial^{2} \psi}}{\partial x \partial y} \\
\frac{\partial \psi}{\partial t} & =-2 \nu \frac{\partial^{2} \phi}{\partial x \partial y}+2 \nu \frac{\overline{\partial^{2} \psi}}{\partial x^{2}}
\end{aligned}
$$

where use has been made of $\boldsymbol{v}=\boldsymbol{\nabla} \phi+\boldsymbol{\nabla} \times \psi \boldsymbol{k}$. 
As these differential equations have constant coefficients, the stability analysis need only make use of the Fourier modes, given by

$$
\begin{aligned}
\eta_{k}(x, t) & =\alpha_{k}(t) e^{i k x}, \\
\phi_{k}(x, y, t) & =\beta_{k}(t) e^{i k x+k y}, \\
\psi_{k}(x, y, t) & =-i \xi_{k}(t) f_{k}(y) e^{i k x},
\end{aligned}
$$

for each wavenumber $k$. These modes satisfy the conditions of fluid incompressibility and no motion infinitely deep in the fluid. Observe that the exact form of $f_{k}(y)$ is not specified, so that the term $2 \nu \frac{\partial^{2} \psi}{\partial x \partial y}$ in equation (41) cannot be evaluated directly. However, it is shown in Section 6.2 that $\tilde{u}^{r}=\mathcal{O}\left(\epsilon^{2}\right)$, and so the problematic term can be neglected, since $\frac{\overline{\partial^{2} \psi}}{\partial x \partial y}=\mathcal{O}\left(\frac{\partial \tilde{u}^{r}}{\partial x}\right)=\mathcal{O}\left(\epsilon^{2}\right)$, i.e. $f_{k}^{\prime}(0)=\mathcal{O}(\epsilon)$. Substituting the Fourier components into equations (40)(42), and equating coefficients, then gives the linear system

$$
\left[\begin{array}{c}
\dot{\alpha}_{k} \\
\dot{\beta}_{k} \\
\dot{\xi}_{k}
\end{array}\right]=\left[\begin{array}{ccc}
0 & k & -k \\
-g-\varsigma k^{2} & -2 \nu k^{2} & 0 \\
0 & 2 \nu k^{2} & -2 \nu k^{2}
\end{array}\right]\left[\begin{array}{c}
\alpha_{k} \\
\beta_{k} \\
\xi_{k}
\end{array}\right],
$$

which has eigenvalues

$$
\lambda_{k}=0,-2 \nu k^{2} \pm i \sqrt{k\left(g+\varsigma k^{2}\right)} .
$$

The non-zero eigenvalues correspond to waves dissipating due to viscosity. Thus, our model of weakly vortical flow is consistent with the physical properties of viscous fluids. The marginal eigenvalues $\lambda_{k}=0$ indicate the presence 
of a centre manifold of small-amplitude, nearly-stationary disturbances. A more detailed nonlinear analysis is required to investigate the true dynamics of such waves.

In contrast, the weakly vortical model of Lundgren [3], written in twodimensional form, is

$$
\begin{aligned}
\frac{\partial \eta}{\partial t} & =\left(\tilde{v}-\frac{\widetilde{\partial \psi}}{\partial s}\right)\left[1+\left(\frac{\partial \eta}{\partial x}\right)^{2}\right]^{-1 / 2}, \\
\frac{d \tilde{\phi}}{d t} & =-g \eta+\frac{1}{2}\left(\tilde{u}^{2}+\tilde{v}^{2}\right)-2 \frac{\partial v}{\partial s} \tilde{\psi}-2 \nu \frac{\partial v}{\partial n}+\varsigma \kappa, \\
\frac{d \tilde{\psi}}{d t} & =2 \frac{\partial v}{\partial n} \tilde{\psi}-2 \nu \frac{\tilde{\partial v}}{\partial s},
\end{aligned}
$$

where the applied shear stress has been neglected. The corresponding linear system is

$$
\left[\begin{array}{c}
\dot{\alpha}_{k} \\
\dot{\beta}_{k} \\
\dot{\xi}_{k}
\end{array}\right]=\left[\begin{array}{ccc}
0 & k & -k \\
-g-\varsigma k^{2} & -2 \nu k^{2} & 0 \\
0 & 2 \nu k^{2} & 0
\end{array}\right]\left[\begin{array}{c}
\alpha_{k} \\
\beta_{k} \\
\xi_{k}
\end{array}\right]
$$

and the stability of this model is therefore governed by

$$
\lambda_{k}^{3}+2 \nu k^{2} \lambda_{k}^{2}+k\left(g+\varsigma k^{2}\right) \lambda_{k}-2 \nu k^{3}\left(g+\varsigma k^{2}\right)=0 .
$$

For small values of viscosity $\nu$, the eigenvalues are

$$
\lambda_{k}=2 k^{2} \nu-\frac{16 k^{5} \nu^{3}}{g+\varsigma k^{2}}+\mathcal{O}\left(\nu^{5}\right)
$$


and

$$
\lambda_{k}=-2 k^{2} \nu+\frac{8 k^{5} \nu^{3}}{g+\varsigma k^{2}} \pm i\left(1+\frac{2 k^{3} \nu^{2}}{g+\varsigma k^{2}}\right) \sqrt{k\left(g+\varsigma k^{2}\right)}+\mathcal{O}\left(\nu^{4}\right) .
$$

The complex eigenvalues correspond to damped waves with frequencies which are slightly different to the physical undamped waves. The problem lies with the real eigenvalues (53), which indicate that some modes will grow exponentially fast and thus ruin simulations based on the model.

As a further comparison, consider the weakly vortical surface model of Ruvinsky et al. [6], written as

$$
\begin{aligned}
\frac{\partial \eta}{\partial t} & =\widetilde{\partial \phi}+\widetilde{v^{r}}-\epsilon \frac{\partial \eta}{\partial x} \frac{\widetilde{\partial \phi}}{\partial x} \\
c^{2} \frac{\widetilde{\partial \phi}}{\partial t} & =-\eta-\frac{1}{2} \epsilon c^{2}\|\widetilde{\nabla \phi}\|^{2}+\varsigma \frac{\partial^{2} \eta}{\partial x^{2}}\left[1+\left(\epsilon \frac{\partial \eta}{\partial x}\right)^{2}\right]^{-3 / 2}-2 \nu \frac{\widetilde{\partial^{2} \phi}}{\partial y^{2}} \\
c^{2} \frac{\widetilde{\partial v^{r}}}{\partial t} & =2 \nu \frac{\widetilde{\partial^{3} \phi}}{\partial y \partial x^{2}}
\end{aligned}
$$

where $c$ is a non-dimensional constant proportional to the phase velocity of gravity-capillary waves, $v^{r}$ is the vertical component of the vortical velocity, and $\epsilon$ is a small parameter which governs the size of the quadratic terms. Hence, linearisation is achieved by setting $\epsilon=0$, which results in

$$
\left[\begin{array}{c}
\dot{\alpha}_{k} \\
\dot{\beta}_{k} \\
\dot{\xi}_{k}
\end{array}\right]=\frac{1}{c^{2}}\left[\begin{array}{ccc}
0 & c^{2} k & -c^{2} k \\
-g-\varsigma k^{2} & -2 \nu k^{2} & 0 \\
0 & 2 \nu k^{2} & 0
\end{array}\right]\left[\begin{array}{c}
\alpha_{k} \\
\beta_{k} \\
\xi_{k}
\end{array}\right]
$$


The stability of this new system is governed by

$$
\lambda_{k}^{3}+\frac{2 \nu k^{2}}{c^{2}} \lambda_{k}^{2}+\frac{k\left(g+\varsigma k^{2}\right)}{c^{2}} \lambda_{k}-\frac{2 \nu k^{3}\left(g+\varsigma k^{2}\right)}{c^{4}}=0 .
$$

Again expanding in small values of viscosity, the eigenvalues are

$$
\lambda_{k}=\frac{2 k^{2} \nu}{c^{2}}-\frac{16 k^{5} \nu^{3}}{c^{4}\left(g+\varsigma k^{2}\right)}+\mathcal{O}\left(\nu^{5}\right),
$$

and

$$
\begin{aligned}
\lambda_{k}= & -\frac{2 k^{2} \nu}{c^{2}}+\frac{8 k^{5} \nu^{3}}{c^{4}\left(g+\varsigma k^{2}\right)} \\
& \pm i\left(1+\frac{2 k^{3} \nu^{2}}{c^{2}\left(g+\varsigma k^{2}\right)}\right) \sqrt{\frac{k\left(g+\varsigma k^{2}\right)}{c^{2}}}+\mathcal{O}\left(\nu^{4}\right) .
\end{aligned}
$$

Observe from (60) that the model of Ruvinsky et al. [6] therefore has the same type of non-physical, unstable dynamics as the model of Lundgren [3]. In contrast, we have shown that our model is consistent with the dissipation of energy expected of weakly viscous fluids.

\section{$6 \quad$ Numerical velocity calculations}

The Lagrangian model of Section 4 forms the basis of a numerical scheme for integrating the fluid dynamical system. Here we adopt and expand upon the algorithms described in detail by Roberts [5], and Mercer and Roberts [4]. 
In outline, let the spatial and temporal variation of the interface be parametrised by a variable $s$ and time $t$, respectively, and denote any arbitrary quantity evaluated at the interface, say $\tilde{q}=q(x, \eta(x, t), t)$, by its capital letter, say $Q(s, t)$. The relevant interfacial variables are then given by $\tilde{x}=X(s, t), \tilde{y}=Y(s, t), \tilde{\phi}=\Phi(s, t)$ and $\tilde{\psi}=\Psi(s, t)$. We further use an overdot " " to denote $\frac{\partial}{\partial t}$ at fixed $s$, and a prime to denote $\frac{\partial}{\partial s}$ at fixed $t$. The Lagrangian model (30)-(33) then becomes

$$
\begin{aligned}
\dot{X} & =U, \\
\dot{Y} & =V, \\
\dot{\Phi} & =-g Y+\frac{1}{2}\left(U^{2}+V^{2}\right)+\varsigma K-2 \nu T-\Psi W, \\
\dot{\Psi} & =-\nu W,
\end{aligned}
$$

respectively. Observe that the interfacial vorticity is

$$
W=2\left(V^{\prime} X^{\prime}-U^{\prime} Y^{\prime}\right)\left(X^{\prime 2}+Y^{\prime 2}\right)^{-1},
$$

from equation (38); the magnitude of the interfacial normal straining force is

$$
T=-\frac{U^{\prime}}{X^{\prime}}-\frac{V^{\prime}}{X^{\prime}} \frac{Y^{\prime}}{X^{\prime}},
$$

from equation (39); and the interfacial curvature is

$$
K=\left(X^{\prime} Y^{\prime \prime}-Y^{\prime} X^{\prime \prime}\right)\left(X^{\prime 2}+Y^{\prime 2}\right)^{-3 / 2}
$$


from equation (35).

The following two sub-sections describe how to evaluate the irrotational and rotational parts, respectively, of the complex interfacial velocity $\mathcal{V}$. Once both these quantities have been determined, the horizontal and vertical components, $U$ and $V$, are found from $U-i V=\mathcal{V}^{i}+\mathcal{V}^{r}$. The associated variables $W, T$ and $K$ are then computed from equations (66)-(68). Finally, the interface at later times are found by numerically integrating the fluid dynamical equations (62)-(65).

\subsection{Irrotational velocity calculations}

The fluid flow is approximately irrotational due to the assumption of weak vorticity. Hence, the complex velocity $\mathcal{V}=U-i V$ is decomposed as $\mathcal{V}=$ $\mathcal{V}^{i}+\mathcal{V}^{r}$, where $\mathcal{V}^{i}$ is the irrotational part and $\mathcal{V}^{r}$ is the rotational or vortical part, with $\mathcal{V}^{r} \ll \mathcal{V}^{i}$. This so-called quasi-irrotational flow means that $\mathcal{V}$ is modelled principally by a vortex sheet along the interface (Van de Vooren [8]), with an additional small correction for vorticity. Following Roberts [5], the vortex sheet engenders the complex velocity potential function

$$
f(z)=\phi+i \psi_{c}=-\frac{i}{2 \pi} \int_{-\infty}^{\infty} a(\sigma, t) \log [z-Z(\sigma, t)] d \sigma
$$

where $z=x+i y, Z=X+i Y, \psi_{c}$ is a harmonic function conjugate to $\phi$ (and not the vortical potential $\psi$ ), and $a(s, t)$ is the vortex strength at the 
point on the interface parametrised by $s$. The infinite integral is replaced by a finite integral by assuming $N$-periodic flow. The irrotational velocity $\mathcal{V}^{i}$ of the lower fluid at a point $Z=Z(s, t)$ on the interface is then

$$
\mathcal{V}^{i}=f^{\prime}(Z)=-\frac{i}{4 \pi} \oint_{0}^{N} a(\sigma, t) \cot \left[\frac{Z-Z(\sigma, t)}{2}\right] d \sigma+\frac{a}{2 Z^{\prime}} .
$$

where use has been made of the Mittag-Leffler expansion (Spiegel [7]), and the Cauchy principal value integral (Spiegel [7]). The complex velocity $\mathcal{V}^{i}$ is related directly to the velocity potential $\Phi$ via

$$
\Phi^{\prime}+i \Psi_{c}^{\prime}=\frac{\partial f(Z)}{\partial s}=f^{\prime}(Z) Z^{\prime}=\mathcal{V}^{i} Z^{\prime},
$$

since $\widetilde{f(z)}=f(Z)=\Phi+i \Psi_{c}$ from equation (69). Hence, multiplying equation $(70)$ by $Z^{\prime}$ and taking the real part gives

$$
\Phi^{\prime}=\frac{1}{4 \pi} \oint_{0}^{N} a(\sigma, t) \Im\left\{Z^{\prime} \cot \left[\frac{Z-Z(\sigma, t)}{2}\right]\right\} d \sigma+\frac{a}{2} .
$$

In order to make the numerical model tractable, the continuous interface is discretised into $N$ points, parametrised by replacing $s \in[0, N)$ by $k=$ $0,1, \ldots, N-1$. Observe that $k=N$ is equivalent to $k=0$ for an $N$-periodic interface. The integrals are then approximated by summations over these grid-points, replacing $\sigma$ by $j=0,1, \ldots, N-1$. However, observe that the integrands in both equations (70) and (72) become singular at $Z=Z(k, t)$ 
whenever $\sigma=k$. It was shown by Roberts [5] that these singularities are removable, giving

$$
\mathcal{V}_{k}=-\frac{i}{4 \pi} \sum_{\substack{j=0 \\ j \neq k}}^{N-1} a_{j} \cot \left[\frac{Z_{k}-Z_{j}}{2}\right]+\frac{a_{k}}{2 Z_{k}^{\prime}}-\frac{i}{4 \pi}\left\{\frac{a_{k} Z_{k}^{\prime \prime}}{Z_{k}^{\prime 2}}-\frac{2 a_{k}^{\prime}}{Z_{k}^{\prime}}\right\},
$$

and

$$
\Phi_{k}^{\prime}=\frac{1}{4 \pi} \sum_{\substack{j=0 \\ j \neq k}}^{N-1} a_{j} \Im\left\{Z_{k}^{\prime} \cot \left[\frac{Z_{k}-Z_{j}}{2}\right]\right\}+\frac{a_{k}}{2}+\frac{1}{4 \pi} \Im\left\{\frac{a_{k} Z_{k}^{\prime \prime}}{Z_{k}^{\prime}}\right\},
$$

where $Z_{k}$ denotes $Z(k, t)$, etcetera. Hence, the irrotational velocities at the interface are now calculated by:

1. using a discrete Fourier transform (FFT) to efficiently and accurately compute the spatial derivatives $Z_{k}^{\prime}, Z_{k}^{\prime \prime}$, and $\Phi_{k}^{\prime}$;

2. solving the linear system (74) for $k=0,1, \ldots, N-1$ to find the vortex strengths $a_{k}(t)$;

3. using an FFT to calculate $a_{k}^{\prime}$;

4. evaluating equation (73) to find the irrotational velocities $\mathcal{V}_{k}^{i}$.

The above approach allows the irrotational velocities on the interface to be uniquely determined. 


\subsection{Vortical velocity calculations}

Recall from the previous section that the vortical flow, governed by the potential $\Psi$, is weak in comparison to the irrotational flow. That is, the complex vortical velocity $\mathcal{V}^{r}$ is small, i.e. $\mathcal{V}^{r} \ll \mathcal{V}^{i}$. In order to derive a formula for the numerical calculation of $\mathcal{V}^{r}$, first consider the theoretical model of Section 3.

Observe, from the chain rule, that

$$
\frac{\partial \tilde{\psi}}{\partial x}=\frac{\widetilde{\partial \psi}}{\partial x}+\widetilde{\frac{\partial \psi}{\partial y}} \frac{\partial \eta}{\partial x}=-\tilde{v}^{r}+\tilde{u}^{r} \frac{\partial \eta}{\partial x},
$$

where $u^{r}=\frac{\partial \psi}{\partial y}$ and $v^{r}=-\frac{\partial \psi}{\partial x}$ from equation (9). Now observe that the normal component of the vortical velocity is

$$
\tilde{\boldsymbol{v}}^{r} \cdot \boldsymbol{n}=\left(\tilde{u}^{r} \boldsymbol{i}+\tilde{v}^{r} \boldsymbol{j}\right) \cdot \frac{1}{S}\left(-\frac{\partial \eta}{\partial x} \boldsymbol{i}+\boldsymbol{j}\right)=\frac{1}{S}\left(\tilde{v}^{r}-\tilde{u}^{r} \frac{\partial \eta}{\partial x}\right),
$$

where, for convenience,

$$
S=\sqrt{1+\left(\frac{\partial \eta}{\partial x}\right)^{2}}
$$

Hence, we deduce that

$$
\tilde{\boldsymbol{v}}^{r} \cdot \boldsymbol{n}=-\frac{1}{S} \frac{\partial \tilde{\psi}}{\partial x}
$$


Similarly, the tangential component of the vortical velocity is

$$
\tilde{\boldsymbol{v}}^{r} \cdot \boldsymbol{s}=\left(\tilde{u}^{r} \boldsymbol{i}+\tilde{v}^{r} \boldsymbol{j}\right) \cdot \frac{1}{S}\left(\boldsymbol{i}+\frac{\partial \eta}{\partial x} \boldsymbol{j}\right)=\frac{1}{S}\left(\tilde{u}^{r}+\tilde{v}^{r} \frac{\partial \eta}{\partial x}\right) .
$$

In contrast to the normal component, the tangential component does not obey a physically-based condition such as (76). We are therefore free to arbitrarily assign such a condition to ensure unique solutions for $\tilde{u}^{r}$ and $\tilde{v}^{r}$; for simplicity, set

$$
\tilde{\boldsymbol{v}}^{r} \cdot \boldsymbol{s}=0
$$

Simultaneously solving (78) and (80) for $\tilde{u}^{r}$ and $\tilde{v}^{r}$ then gives

$$
\tilde{u}^{r}=\frac{1}{S^{2}} \frac{\partial \tilde{\psi}}{\partial x} \frac{\partial \eta}{\partial x}, \quad \tilde{v}^{r}=-\frac{1}{S^{2}} \frac{\partial \tilde{\psi}}{\partial x} .
$$

Observe that small perturbations of size $\tilde{\psi}, \eta=\mathcal{O}(\epsilon)$ have the consequence that $\tilde{u}^{r}=\mathcal{O}\left(\epsilon^{2}\right)$ and $\tilde{v}^{r}=\mathcal{O}(\epsilon)$. This property was used in Section 5 to eliminate the term $\frac{\partial u^{r}}{\partial x}$ from the perturbation model, which retained only terms linear in $\mathcal{O}(\epsilon)$.

Finally, in terms of the numerical model, observe that the vortical velocity components become

$$
U^{r}=\frac{\Psi^{\prime} Y^{\prime}}{X^{\prime 2}+Y^{\prime 2}}, \quad V^{r}=-\frac{\Psi^{\prime} X^{\prime}}{X^{\prime 2}+Y^{\prime 2}},
$$


respectively. Hence, the complex vortical velocity $\mathcal{V}^{r}$ is

$$
\mathcal{V}^{r}=U^{r}-i V^{r}=\frac{i \Psi^{\prime} Z^{\prime *}}{Z^{\prime} Z^{\prime *}},
$$

where $Z^{\prime}=X^{\prime}+i Y^{\prime}$, and the asterisk denotes complex conjugation. By simplification and rearrangement, observe that

$$
i \Psi^{\prime}=\mathcal{V}^{r} Z^{\prime}
$$

which is similar in form to equation (71), viz

$$
\Phi^{\prime}+i \Psi_{c}^{\prime}=\mathcal{V}^{i} Z^{\prime}
$$

for the complex irrotational velocity.

\section{$7 \quad$ Numerical verification of the model}

The numerical model of the previous section predicts the time-evolution of fluid quantities evaluated at discrete points on the interface with reasonable accuracy and efficiency. A fourth-order Runge-Kutta scheme is used here to integrate the numerical model. We verify our earlier theoretical prediction that small-amplitude wave perturbations to a flat interface exhibit only conservative or dissipative fluid dynamics.

For this simulation, walls are imposed at $x=0$ and $\pi$, corresponding to the discrete points $k=0$ and $k=M=N / 2$ along the interface; hence, 
$X_{0}=0$ and $X_{M}=\pi$, respectively. Assuming the fluid remains in constant contact with the walls, i.e. there is no cavitation within the fluid at the walls, then the necessary boundary conditions are

$$
u=0 \quad \text { at } x=0, \pi \Rightarrow U_{0}=U_{M}=0,
$$

The required $N$-periodicity of the interface is now ensured by reflectional symmetry about $k=M$ and $k=0$, such that

$$
\begin{aligned}
X_{N-k}=2 \pi-X_{k}, & Y_{N-k}=Y_{k}, \\
\Phi_{N-k}=\Phi_{k}, & \Psi_{N-k}=-\Psi_{k}, \\
U_{N-k}=-U_{k}, & V_{N-k}=V_{k},
\end{aligned}
$$

for $k=0,1, \ldots, M$. The short-term stability of the interface is now determined by computing the eigenvalues of the approximate linearisation matrix, obtained from the time-derivative routine of the numerical model using small perturbations to a flat interface. Here we use a total of $N=16$ discrete points to represent the symmetrically reflected fluid. The sawtooth instability is eliminated, as per Roberts [5], by temporarily doubling the number of discrete points along the interface in the velocity calculations, and zeroing the highest Fourier coefficient.

Table 1 shows the eigenvalues computed for purely inviscid gravity waves, in comparison to the corresponding theoretical values obtained from the perturbation expansions of Section 5. Overall there is excellent agreement. The fact that the numerical model exhibits a non-zero eigenvalue $\lambda_{0}$ is due to an 
TABLE 1: Eigenvalues (using $N=16$ ) for small-amplitude, free interfacial waves $(\nu=0, \varsigma=0)$, verifying that $\lambda_{k}= \pm i \sqrt{g k}$ to numerical accuracy.

\begin{tabular}{|c|cc||cc|}
\hline & \multicolumn{2}{|c||}{ Theoretical Model } & \multicolumn{2}{c|}{ Numerical Model } \\
\hline$k$ & $\Re\left(\lambda_{k}\right)$ & $\Im\left(\lambda_{k}\right)$ & $\Re\left(\lambda_{k}\right)$ & $\Im\left(\lambda_{k}\right)$ \\
\hline 0 & 0.00000000 & 0.00000000 & 0.00000019 & 0.00000000 \\
1 & 0.00000000 & 1.00000000 & 0.00000008 & 1.00000000 \\
2 & 0.00000000 & 1.41421356 & 0.00000007 & 1.41421356 \\
3 & 0.00000000 & 1.73205081 & 0.00000005 & 1.73205081 \\
4 & 0.00000000 & 2.00000000 & 0.00000004 & 2.00000000 \\
5 & 0.00000000 & 2.23606798 & 0.00000002 & 2.23606798 \\
6 & 0.00000000 & 2.44948974 & 0.00000001 & 2.44948975 \\
7 & 0.00000000 & 2.64575131 & 0.00000000 & 2.64575131 \\
8 & 0.00000000 & 2.82842712 & 0.00000000 & 2.82842713 \\
\hline
\end{tabular}

insignificant departure from incompressibility of the liquid, which is explained later. That the other eigenvalues have slightly positive real-parts is attributed to the use of numerical finite differences for derivatives. Similarly, Table 2 shows the eigenvalues obtained for inviscid capillary-gravity waves. Again there is excellent agreement with the theoretical eigenvalues (47). Lastly, Table 3 shows the eigenvalues obtained for weakly vortical gravity waves, which are again in excellent agreement with the theoretical eigenvalues (47). Observe that the numerical damping rate of wavenumber $k=8$, given by the real part of $\lambda_{8}$, is half the theoretical rate. This is most likely an artefact of the way we suppress the sawtooth instability. 
TABLE 2: Eigenvalues (using $N=16)$ for inviscid $(\nu=0)$ capillary-gravity waves, verifying that $\lambda_{k}= \pm i \sqrt{k\left(g+\varsigma k^{2}\right)}$ to numerical accuracy.

\begin{tabular}{|r|rr||rr|}
\hline & \multicolumn{2}{|c||}{ Theoretical Model } & \multicolumn{2}{c|}{ Numerical Model } \\
\hline$k$ & $\Re\left(\lambda_{k}\right)$ & $\Im\left(\lambda_{k}\right)$ & $\Re\left(\lambda_{k}\right)$ & $\Im\left(\lambda_{k}\right)$ \\
\hline 0 & 0.00000000 & 0.00000000 & 0.00000019 & 0.00000000 \\
1 & 0.00000000 & 1.15022047 & 0.00000008 & 1.15022045 \\
2 & 0.00000000 & 2.14104111 & 0.00000007 & 2.14104109 \\
3 & 0.00000000 & 3.42362272 & 0.00000005 & 3.42362270 \\
4 & 0.00000000 & 4.96713764 & 0.00000004 & 4.96713765 \\
5 & 0.00000000 & 6.73616295 & 0.00000002 & 6.73616295 \\
6 & 0.00000000 & 8.70457007 & 0.00000001 & 8.70457008 \\
7 & 0.00000000 & 10.85317675 & 0.00000000 & 10.85317677 \\
8 & 0.00000000 & 13.16736620 & 0.00000000 & 13.16737070 \\
\hline
\end{tabular}


TABLE 3: Eigenvalues (using $N=16$ ) for weakly vortical gravity ( $\varsigma=0$ ) waves, verifying that $\lambda_{k}=-2 \nu k^{2} \pm i \sqrt{g k}$ to numerical accuracy.

\begin{tabular}{|r|rr||rr|}
\hline & \multicolumn{2}{|c||}{ Theoretical Model } & \multicolumn{2}{c|}{ Numerical Model } \\
\hline$k$ & $\Re\left(\lambda_{k}\right)$ & $\Im\left(\lambda_{k}\right)$ & $\Re\left(\lambda_{k}\right)$ & $\Im\left(\lambda_{k}\right)$ \\
\hline 0 & 0.00000000 & 0.00000000 & 0.00000019 & 0.00000000 \\
1 & -0.00172853 & 1.00000000 & -0.00172845 & 1.00000000 \\
2 & -0.00691412 & 1.41421356 & -0.00691406 & 1.41421356 \\
3 & -0.01555677 & 1.73205081 & -0.01555672 & 1.73205081 \\
4 & -0.02765649 & 2.00000000 & -0.02765645 & 2.00000000 \\
5 & -0.04321326 & 2.23606798 & -0.04321324 & 2.23606798 \\
6 & -0.06222709 & 2.44948974 & -0.06222708 & 2.44948975 \\
7 & -0.08469799 & 2.64575131 & -0.08469798 & 2.64575131 \\
8 & -0.11062592 & 2.82842712 & -0.05531297 & 2.82788622 \\
\hline
\end{tabular}


On a longer time-scale, the validity of the numerical model is verified by computing various quantities which theoretically are invariant. Roberts [5] defines four such quantities, namely: the mean vertical displacement of the interface, $C$; the average volume flux across the interface, $\Omega$; the average horizontal momentum, $I$; and the total interfacial energy $E$. The initial interfacial profile is set to a single wave $y=A \cos x$ of amplitude $A=0.125$. The evaluations of the physical invariants are shown in Figure 1, obtained for inviscid gravity flow. Observe that the average volume flux $\Omega$, shown in Figure $1(\mathrm{~b})$, is a numerically invariant quantity, apart from random truncation errors of size $10^{-13}$. Similarly, the average horizontal momentum $I$, shown in Figure 1(c), is numerically invariant to within an error of size $10^{-18}$. Observe, however, that the mean interfacial position $C$, shown in Figure 1(a), is increasing overall with time, with a slope of about $10^{-11}$. This indicates that the numerical model has a negligible departure from strict incompressibility, which was revealed in Table 1 by the occurrence of a non-zero eigenvalue for wavenumber $k=0$. The slight lack of invariance for $C$ is a direct consequence of using a discrete integration scheme to approximate the continuous temporal dynamics of the fluid. This same problem also affects the total energy $E$, which slowly decreases with time, as shown in Figure 1(d). Since slope of the line is only about $10^{-9}$, however, the total energy is effectively conserved over this time-scale. 
(a) Mean interfacial height $C$ versus time $t$

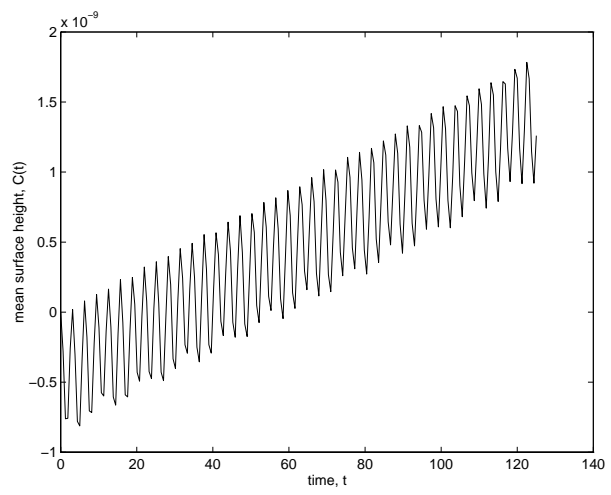

(b) Average volume flux $\Omega$ versus time $t$

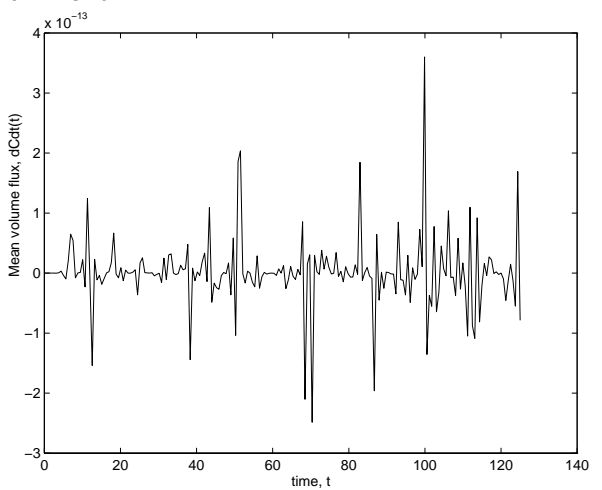

FIGURE 1: Numerical estimates of four physically invariant quantities, showing that both the average volume flux $\Omega$, (b), and the average horizontal momentum $I,(\mathrm{c})$, are approximately invariant. The mean displacement $C$, (a), is slowly increasing with time, implying a slight lack of incompressibility of the fluid. The total energy $E,(d)$, slowly decreases with time, indicating a slight dissipation of energy in the numerically evaluated fluid dynamical system. 
(c) Average horizontal momentum $I$ versus time $t$

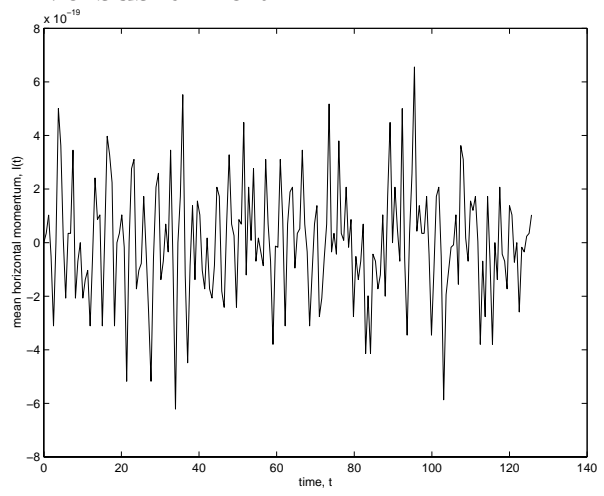

(d) Change in total interfacial energy, $E-E_{0}$, versus time $t$

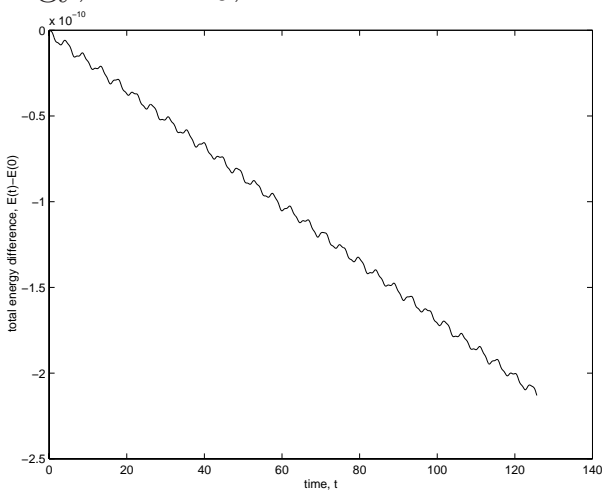

Figure 1: continued 


\section{Conclusion}

We have derived a boundary-integral model to describe the two-dimensional dynamics of a weakly viscous, quasi-irrotational fluid at the interface with a light, dynamically inactive fluid. Vortical flow is restricted to a thin boundary layer near the interface. This model has the important property of exhibiting only conservative and dissipative modes of linear behaviour for smallamplitude interfacial waves. In contrast, we have shown that comparable models of weak vorticity (Lundgren [3], Ruvinsky et al. [6]) exhibit exponentially growing non-physical modes.

Numerical simulations of the interfacial fluid dynamics depended chiefly on a vortex sheet approximation to the irrotational fluid flow (Roberts [5]), which enabled the stable and accurate computation of interfacial fluid velocities. The expected behaviour of the model was verified by numerical analysis of fundamental wave modes, and by the appropriate conservation of key physical invariants.

\section{References}

[1] Batchelor, G.K. (1967): An introduction to fluid dynamics. Cambridge University Press. E73 
[2] Holmes, P. (1986): Chaotic motions in a weakly nonlinear model for surface waves. J. Fluid Mech., 162, 365-388. E71

[3] Lundgren, T.S. (1989): A free surface vortex method with weak viscous effects. Mathematical Aspects of Vortex Dynamics (ed. R.E. Caflisch), SIAM, Philadelphia, 68-79. E71, E71, E72, E77, E80, E83, E85, E100

[4] Mercer, G.N. and Roberts, A.J. (1992): Standing waves in deep water; their stability and extreme form. Phys. Fluids A, 4, 259-269. E85

[5] Roberts, A.J. (1983): A stable and accurate numerical method to calculate the motion of a sharp interface between fluids. IMA J. App. Math., 31, 13-35. E85, E87, E89, E93, E97, E100

[6] Ruvinsky, K.D., Feldstein, F.I. and Freidman, G.I. (1991): Numerical simulations of the quasi-stationary stage of ripple excitation by steep gravity-capillary waves. J. Fluid Mech., 230, 339-353. E72, E80, E84, E85, E100

[7] Spiegel, M.R. (1964): Theory and problems of complex variables. Schaum's Outline Series, McGraw-Hill Book Company. E88, E88

[8] Van de Vooren, A.I. (1980): A numerical investigation of the rolling-up of vortex sheets. Proc. R. Soc. Lond. A, 373, 67-91. E71, E87 